Quote of the Day Protocol

This RFC specifies a standard for the ARPA Internet community. Hosts on the ARPA Internet that choose to implement a Quote of the Day Protocol are expected to adopt and implement this standard.

A useful debugging and measurement tool is a quote of the day service. A quote of the day service simply sends a short message without regard to the input.

TCP Based Character Generator Service

One quote of the day service is defined as a connection based application on TCP. A server listens for TCP connections on TCP port 17. Once a connection is established a short message is sent out the connection (and any data received is thrown away). The service closes the connection after sending the quote.

UDP Based Character Generator Service

Another quote of the day service is defined as a datagram based application on UDP. A server listens for UDP datagrams on UDP port 17. When a datagram is received, an answering datagram is sent containing a quote (the data in the received datagram is ignored).

\title{
Quote Syntax
}

There is no specific syntax for the quote. It is recommended that it be limited to the ASCII printing characters, space, carriage return, and line feed. The quote may be just one or up to several lines, but it should be less than 512 characters. 